\title{
BMJ Open Mangomoments - preconditions and impact on patients and families, healthcare professionals and organisations: a multi-method study in Flemish hospitals
}

To cite: Vanhaecht $K$, Van Bael E, Coeckelberghs E, et al. Mangomoments preconditions and impact on patients and families, healthcare professionals and organisations: a multi-method study in Flemish hospitals. BMJ Open 2020;10:e034543. doi:10.1136/ bmjopen-2019-034543

- Prepublication history and additional material for this paper are available online. To view these files, please visit the journal online (http://dx.doi org/10.1136/bmjopen-2019034543).

Received 25 September 2019 Revised 02 July 2020

Accepted 08 July 2020

Check for updates

(C) Author(s) (or their employer(s)) 2020. Re-use permitted under CC BY-NC. No commercial re-use. See rights and permissions. Published by BMJ.

For numbered affiliations see end of article.

Correspondence to

Dr Deborah Seys;

Deborah.seys@kuleuven.be

Kris Vanhaecht (D) , ${ }^{1,2}$ Elly Van Bael, ${ }^{1,3}$ Ellen Coeckelberghs, ${ }^{1}$ Charlotte Van der Auwera, ${ }^{1,4}$ Fien Claessens, ${ }^{1}$ Luk Bruyneel (D) , ${ }^{1}$ Dirk De Ridder, ${ }^{1,5}$ Massimiliano Panella, ${ }^{6}$ Maureen Bisognano, ${ }^{7}$ Pedro L Delgado, ${ }^{7}$ Deborah Seys (1) ${ }^{1,8}$

\section{ABSTRACT}

Objective Understanding how small unexpected acts or gestures by healthcare professionals, known as Mangomoments, are translated into practice, what their preconditions are and what their impact is on patients and families, healthcare professionals and organisations. Design A multi-method design was used based on four phases: (1) A (media)campaign to collect Mangomoment stories $(n=1045)$, of which $94 \%(n=983)$ were defined as Mangomoments; (2) Semi-structured interviews ( $n=120)$; (3) Focus group interviews ( $n=3)$; and (4) A consensus meeting.

Setting Respondents from a hospital and primary care setting.

Participants Patients, family, healthcare professionals, managers, researchers and a policymaker participated. Results Mangomoments are mainly classified in the dimensions 'Respect for values, preferences and needs' and 'Emotional support'. Differences in importance of the dimensions were found between healthcare professionals, oncological patients and family and non-oncological patients and family. The results of the interviews, focus groups and consensus meeting were visualised by the Mangomoment model. It identifies several preconditions on the level of patients, healthcare professionals and leadership. For each of these preconditions a catalyst was identified to increase the prevalence of Mangomoments. In general, Mangomoments improved the patient and family experience and facilitated adherence to therapy and led to a positive perception on the healing process. Positive effects for professionals include personal accomplishment and anti-burnout, joy in work and a positive team atmosphere. This led to positive resonance by a relationship of trust between the patient and the healthcare professionals, feelings of tolerance during negative experiences and open communication and a safe climate. Overall, patients and healthcare workers concluded that Mangomoments led to loyalty to the healthcare organisation.

Conclusion Mangomoments do not only have a positive impact on patient and family but also on the
Strengths and limitations of this study

By using a multi-method study, an under investigated area in the field of patient safety and quality of care was explored: positive moments in healthcare and its impact on patient, family and healthcare professionals.

- Based on 120 interviews, 3 focus groups and 1 consensus meeting, a framework for Mangomoments was developed.

- Peer debriefing, data and investigator triangulation promote the validity and credibility of the study, but a member check was not conducted.

- A heterogeneous sample of family, patients, healthcare professionals, management and healthcare policymakers shared a large number of positive stories that helped define Mangomoments, but further research must include neutral and opposite views towards this concept.

- Mangomoments may be influenced by culture, therefore additional studies in other settings should be initiated to understand possible differences between various types of patient groups.

healthcare professional. Leadership should shape several preconditions and catalysts which can lead to positive resonance and loyalty of patients and professionals.

\section{INTRODUCTION}

Healthcare quality and safety research mainly focusses on negative aspects. Recently the Safety II concept was introduced to shift the focus to learning from positive processes. ${ }^{1}$ Attention should not only be given to positive processes to make healthcare safer, but maybe we also need to further understand the impact of positive experiences such as a hug, laugh or even a small moment of positive 


\section{Phase I: \\ - How is the Mangomoment concept translated into practice?}

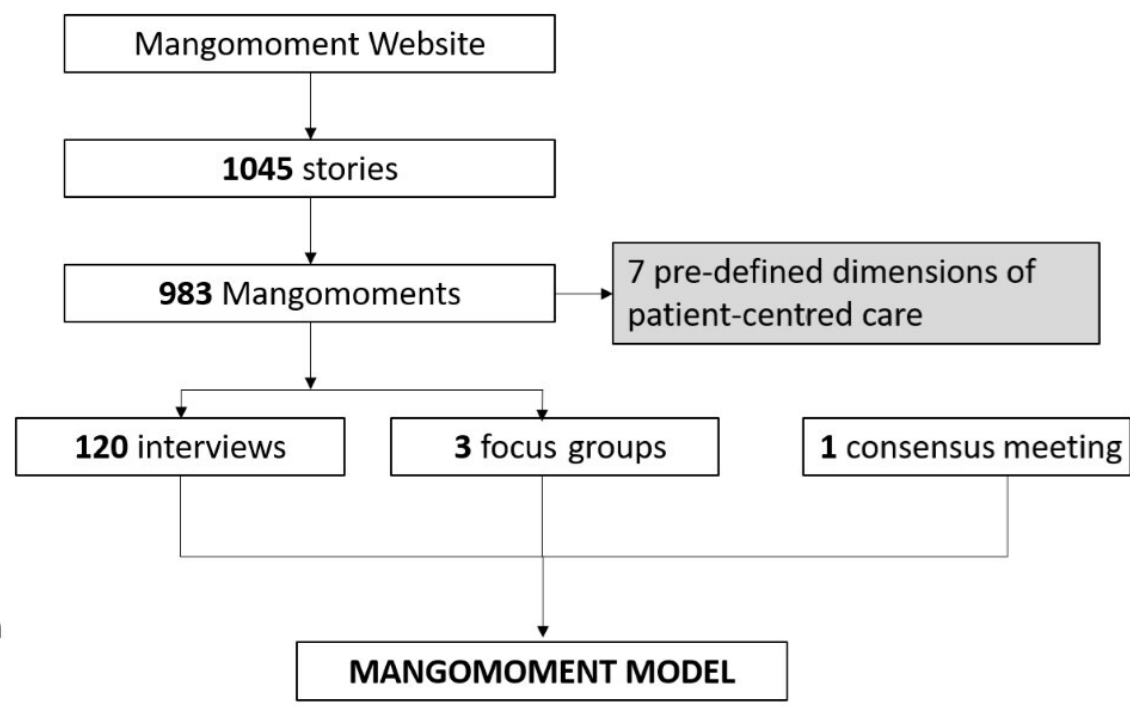

\section{Phase II:}

- What are the preconditions needed to establish Mangomoments?

- What is the impact of Mangomoments on patients, family and healthcare professionals?

\section{MANGOMOMENT MODEL}

Figure 1 Overview of the research project.

emotion. These micromoments of positive resonance, as defined by Fredrickson (2013) and Pronovost (2016) might give people the essential daily nutrients besides healthy food. ${ }^{23}$ In 2018 the Mangomoment concept was introduced and was defined as 'small unexpected acts or gestures, which are of great value in the care experience of patients, residents, families and healthcare professionals. They take place during normal care activities and require nearly no additional resources, time or energy." Mangomoments are different from events like Make-a-Wish or visits by celebrities to children's hospitals. ${ }^{4}$ Although the concept has been defined and is taken to heart by many healthcare organisations, the impact stays unclear.

In this study we want to (1) understand how the Mangomoment concept is translated into practice. Next, we want to (2) understand what preconditions are needed to establish Mangomoments and (3) evaluate the impact of Mangomoments on patients, their family and on healthcare professionals.

\section{METHODS}

\section{Participant recruitment and data collection}

A four phase multi-method design was used in a multicentre setting. Figure 1 demonstrates an overview of the research project. The first part of this study is related to understand how the Mangomoment concept is translated into practice. Therefore, a media campaign was launched and Mangomoments were collected via the Mangomoment website (www.mangomoment.org). Respondents could share their Mangomoment story anonymous or share their demographic data (type of organisation, Mangomoment shared by patient/family or healthcare professional, gender, year of birth, level of education, professional status, home status, oncological care setting or not). They could also mention if they agreed to be contacted for an interview and/or next steps of the study. Data were collected between March 2017 and August
2018. Stories were not selected if they were unclear and included limited information or when the act or gesture was not possible during normal care activities (for example, organising a wedding for a palliative patient).

The second part of our research is related to (1) understanding the preconditions of Mangomoments and (2) evaluating the impact of Mangomoments on patients, family and healthcare professionals. This part contains three phases. In the first phase, respondents who shared a Mangomoment were contacted. Inclusion criteria were, based on information from phase one: (1) gave written consent to be interviewed, (2) shared all demographic variables and (3) submitted a story that met the definition of a Mangomoment. Respondents were then selected purposefully in order to enhance information richness. Cases with a wide range of variation were sought: variation in demographics, variation in dimensions of patientcentred care and information-rich cases. Interviews were performed with patients, family and healthcare professionals in both oncological and non-oncological care processes in hospital setting and primary care. Data were collected and audio-recorded between August 2017 and August 2018 via semi-structured interviews in person or by video call using Skype or FaceTime. Interviews in person took place at a location chosen by the respondent. The interview guide consisted of open-ended questions covering following topics: (1) Why did that specific Mangomoment give you a good feeling, why did it happen?, (2) What was the impact on yourself? (3) What was the impact on the patient-family-professional relation?, and (4) Are there contextual factors or preconditions which will influence the prevalence of Mangomoments? Field notes were taken during the interviews and discussed within the research team. During weekly peer debriefing meetings, saturation was evaluated and there was consensus that 'data saturation' was achieved after 106 interviews since new data repeated what was expressed in previous data. ${ }^{5}$ 
Although saturation was reached, 14 additional interviews were performed as they were already scheduled and the team did not want to disappoint the interviewees. No new insights emerged from that data. In a next phase, three focus groups, each with patients, family and healthcare professionals, were performed in June 2018. A total of 35 persons participated. During these focus groups, the preconditions were further explored. Also the relevance, the feasibility, the facilitators and barriers and the role of management in healthcare organisations was discussed. In the third and last phase, a multi-stakeholder consensus meeting to validate the results of the interviews and focus groups, was performed. This consensus meeting aimed to put the results of the previous phases in a broader context of quality management. The participants to the consensus meeting were patients, family, healthcare professionals, management and healthcare policymakers.

\section{Data analysis}

First, in order to understand how Mangomoments were translated into practice all Mangomoment stories and demographic variables of the respondents were collected in an Excel file. In total 1045 stories were collected of which 983 were defined as Mangomoments. These 983 stories were classified in seven pre-existing categories. These categories are based on the well-known dimensions of patient-centred care which are defined by researchers from Harvard Medical School, on behalf of the Picker Institute and The Commonwealth Fund: (1) respect for patients' values, preferences and expressed needs; (2) emotional support; (3) involvement by family and friends; (4) physical comfort; (5) coordination of care activities; (6) care from volunteers; and (7) information, communication and teaching. ${ }^{6}$ Online supplementary appendix 1 demonstrates examples of Mangomoments per category.

Second, in order to understand the preconditions and impact of Mangomoments 120 interviews, 3 focus groups and a multi-stakeholder consensus meeting were conducted. The interviews and group meetings were audiotaped and both descriptive and reflective field notes were taken. Descriptive notes included observational information about the context and the non-verbal actions. This type of notes contributed to thick description and ensured that the interviewer captured the key information. The reflective notes included methodological notes (eg, thoughts about an approach that did (not) work) and theoretical notes as a starting point for subsequent analysis.

In order to familiarise with the data, audiotapes were listened to by the members of the research team. To ensure investigator triangulation, a team of researchers, experienced researchers and interviewers, was involved. Five experienced researchers, each with a different background, headed the research team. Three of them are full/ associate professors (one of them has clinical experience as a urologist) and two of them hold a MSc degree. All of them are international experts in the field of healthcare quality. The interview analysis team was multidisciplinary and consisted of six persons: three senior researchers (two women and one man, all with a MSc and PhD degree) with experience in healthcare quality, two junior researchers (both women; a nurse and a physiotherapist; both with a MSc degree) and the principal interviewer (woman, MSc degree) with 17 years of clinical experience as a (head) nurse and night supervisor at intensive care in a university hospital. Everyone followed training and had experience with qualitative research. There was no relationship established with interviewees prior to study commencement. Respondents received information about the study in advance. Before the interview the interviewer introduced himself or herself.

Important quotes were transcribed ad verbatim. Field notes and quotes were managed and analysed in NVivo software (QSR international, Durham, UK).

In accordance with the guidelines described by the Qualitative Analysis Guide of Leuven (QUAGOL) method, ${ }^{7}$ open coding was used to identify themes based on the respondents' own words. The coding process consisted of two steps. In the first step paper and pencil were used to develop a list of meaningful concepts. In the second step, the actual coding process took place. Coding software was used to ensure a systematic and repeatable analysis of the concepts based on the data, ending with an empirically based description of the results (which was the basis of the Mangomoment model).

During the data collection period, weekly peer debriefing meetings were organised to discuss field notes and quotes within the research team. Guided by an iterative process, theoretical notes were made and themes and subthemes were defined.

All the respondents gave written informed consent to participate.

\section{Patient and public involvement}

Patients and healthcare professionals were involved in this project.

\section{RESULTS \\ Understanding how Mangomoments are translated into practice}

In total, 1045 stories were collected via the Mangomoment website. Based on the definition $983(94 \%)$ were defined as Mangomoment. They were shared by 620 respondents of which 308 were patients (235 oncological patients and 73 non-oncological patients), 76 were family ( 55 shared a Mangomoment related to an oncological care process) and 236 were healthcare professionals.

Figure 2 demonstrates an overview of the classification of Mangomoments for healthcare professionals, patient/ family for oncological and non-oncological Mangomoments. Mangomoments are mainly classified in the dimensions 'Respect for values, preferences and needs' (38\%) and 'Emotional support' (18\%). The third most common dimension in the group of patients and family is 'Involvement by family and friends' $(15 \%)$. This was higher in 


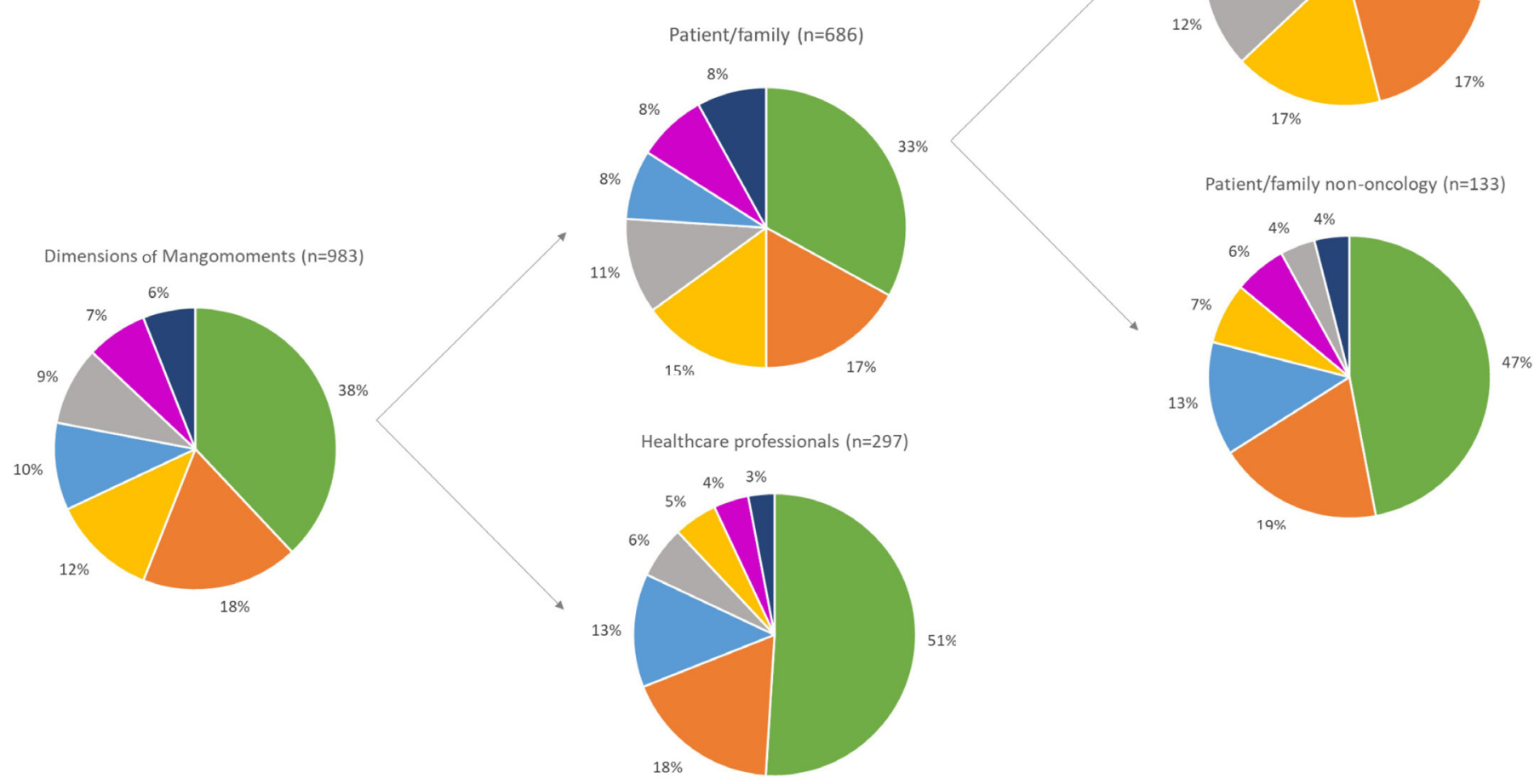

Figure 2 Overview of the dimensions of Mangomoments.

oncological Mangomoments (17\%) compared with nononcological Mangomoments (7\%). While the dimension 'Physical comfort' was higher in non-oncological Mangomoments (13\%) compared with oncological Mangomoments $(7 \%)$. The dimension 'Physical comfort' is also the third most common dimension in the group of the healthcare professionals (13\%). See Online supplementary appendix 1 for examples of Mangomoment stories.

\section{Understanding the preconditions and impact of Mangomoments on patient, family and healthcare professionals: Mangomoment model}

The Mangomoment model is based on the results of 120 semi-structured interviews, 3 focus groups and 1 multistakeholder consensus meeting. Three hundred and fifty-one respondents, who shared a Mangomoment, gave consent to meet the researchers for an interview and 176 respondents were contacted and 120 interviewed (response rate 68\%). Details of the participants in interviews, focus groups and consensus meeting are shown in table 1.

The Mangomoment model consists of several parts: (1) preconditions (figure 3, green), (2) catalysts that lead to Mangomoments (figure 3, blue), (3) the Mangomoment itself, (4) aspects on which the Mangomoments have an impact on (figure 3, yellow) and (5) the overall result (figure 3, red). Online supplementary appendix 2 shows two quotes per construct of the Mangomoment model.

\section{Preconditions}

Based on the data from the interviews and focus groups, it seems like a number of preconditions need to be met before a Mangomoment can be created (see figure 3, green).

\begin{tabular}{|c|c|c|c|}
\hline & $\begin{array}{l}\text { Interviews } \\
(n=120)\end{array}$ & $\begin{array}{l}\text { Focus } \\
\text { groups } \\
(n=35)\end{array}$ & $\begin{array}{l}\text { Consensus } \\
\text { meeting } \\
(n=23)\end{array}$ \\
\hline Patients & 67 & 6 & 4 \\
\hline Family & 18 & 2 & 1 \\
\hline $\begin{array}{l}\text { Healthcare } \\
\text { professionals }\end{array}$ & 35 & 16 & 6 \\
\hline Managers & 0 & 11 & 7 \\
\hline Researchers & 0 & 0 & 4 \\
\hline Policymakers & 0 & 0 & 1 \\
\hline
\end{tabular}




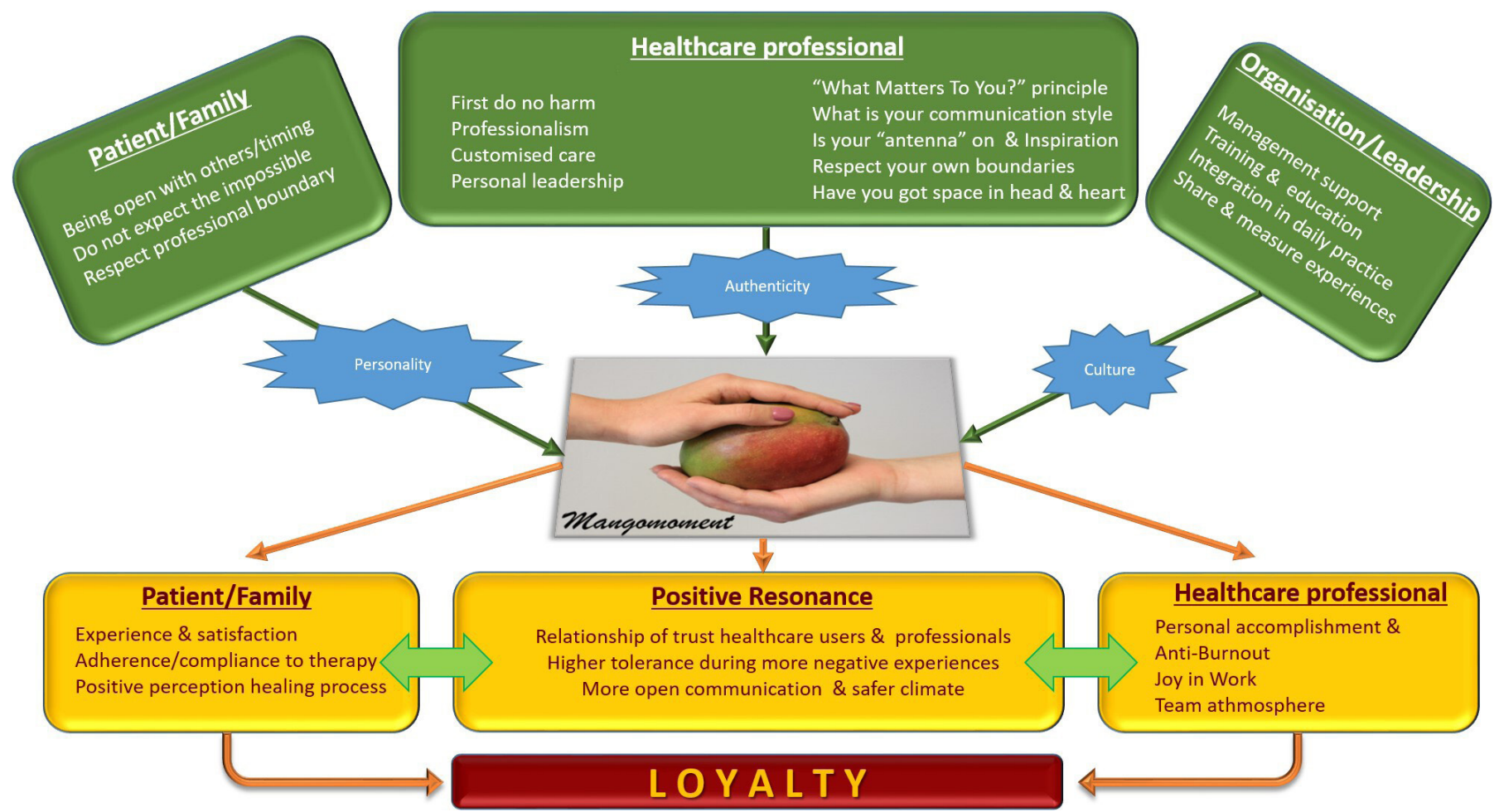

Figure 3 Mangomoment model.

\section{Preconditions for patient/family}

The data revealed that patients and family need to be open to others and aware of the timing. When patients/ families are not open to acts from others, it will be hard to establish a Mangomoment, or it will have less impact. Timing is important as kind gestures will not be appreciated at all times. Next, two types of boundaries emerged from the data. First, there are limitations to what a patient can reasonably 'expect', because a Mangomoment is about small and unexpected things such as a pat on the back or a wink. Second, healthcare professionals seem to be limited by their own professional boundaries, that arise from their own values and norms. Not all healthcare professionals will be able to, or want to, look for a personal connection with a patient or family member. It is important that patients and family respect this.

\section{Preconditions for healthcare professionals}

The data offered insights into the general preconditions that need to be taken into account by the healthcare professional. First, they cannot do harm to a patient as Mangomoments must be in line with safety standards. This might seem obvious, but sometimes a healthcare professional can be tempted to perform an unsafe act to please a patient (eg, giving something to eat to a patient who has not been fed orally for a long time). A second precondition is that healthcare professionals should meet professional requirements, so that they provide excellent clinical care at all times. A third precondition is that the delivered care always has to correspond to the needs, expectations and wishes of the patients. In other words, the care needs to be customised. The fourth precondition is about personal leadership. Healthcare professionals who make the effort to create a Mangomoment are often driven by their personal leadership. Such healthcare professionals can show their clinical leadership as an authentic act to enhance patient-centred care and often model exemplary behaviour within the team.

The data also showed that there are more specific preconditions of which healthcare professionals should be aware. The 'What Matters to You?' (WMTY) principle is suggested as a fifth precondition to offer Mangomoments. WMTY is based on the awareness of the importance to act open-hearted and open-minded in relationship with patients. Knowing what is important to a patient or a family member, for example, by just asking, is of paramount importance. ${ }^{8}$ The sixth precondition is about communication style. The data revealed that both verbal and non-verbal communication need continuous attention so that healthcare professionals can listen carefully and respond appropriately. Seventh, the study showed that one can switch on a 'Mangomoment radar'. At such moments, professionals are open to inspiration from colleagues. Being open to learn from experiences from others can enhance their own Mangomoment capability and creativity. Respect for one's own boundaries to avoid that the healthcare professional himself becomes too personally involved is the eighth precondition. Finally, healthcare professionals will only offer that little extra if they feel good about themselves, or if they have enough space in their hearts and minds. Self-care is not selfish and only healthy healthcare providers will be able to provide authentic Mangomoments. 


\section{Preconditions for organisations/leadership}

For the realisation of Mangomoments, it is important that the healthcare professional and teams have the opportunities to realise them. First, management support seems to be indispensable. The management should support healthcare opportunities and innovations to enhance patient-centred care and adapt care to correspond fully to the needs of patients and family. Second, training and education on patient-centred care and positive resonance (where, for example, Mangomoment stories can be shared) should be part of basic and continuous education so that healthcare professionals can acquire competencies to offer Mangomoments. Third, integration of Mangomoments in daily practice is, according to several respondents, something to strive for. The management and board must set an example and must include Mangomoments in their vision, attitude and behaviour. Finally, according to the data sharing and measuring valuable positive experiences can lead to inspiration and practical advice within teams and organisations to enhance the likelihood of Mangomoments in the daily practice.

\section{Catalysts}

Catalysts act between the preconditions and the Mangomoment itself and are circumstances that increase the likelihood of a Mangomoment (see figure 3, blue).

\section{Personality of patient/family}

The data shows that positive personality traits of the patient or family enhance the willingness of the healthcare provider to do additional things for that patient.

\section{Authenticity of healthcare professional}

The results indicate that the personality and the authenticity of healthcare professionals influence how small acts or gestures are received by patients and their family. This authenticity is evaluated by patients based on the provider's overall attitude, kindness and honesty.

\section{Culture of the organisation}

The organisational culture plays an important role in the realisation of Mangomoments. This is rooted in the values and norms of the teams, leadership and the overall atmosphere in the organisation.

Impact of Mangomoments (see figure 3, Yellow)

\section{On patient/family}

The data showed clearly that Mangomoments lead to a positive experience and satisfaction. They are seen as a moment of pleasure which positively influences patients' view on the provided care. Also, patients tend to feel more motived to follow and comply with the therapy. A Mangomoment can regain the spirit of life and hereby can have a positive perception on the healing process. Several respondents stated that such small gestures gave them the energy to keep going.
Positive resonance

Respondents also indicated a real connection or 'positive resonance' with a healthcare professional who offered them a Mangomoment. Such a positive chemistry leads to a trusting bond and a higher tolerance during more negative experiences in the care relation. Also a more open communication, for example, about doubts and trust, enhances the psychological safety and safety climate.

\section{On healthcare professional}

Respondents indicated that Mangomoments can have a positive effect on their work and experience. According to the data, Mangomoments might lead to personal accomplishment and anti-burnout. Healthcare professional derive satisfaction from their job, which results in more positive energy. This helps them to focus on their work with greater passion. Also joy in work was positively influenced through the creation of Mangomoments. Mangomoments gave professionals a good feeling and an added value to their job (eg, 'This is what we do it for' or 'This goes right to the heart of why we opted for a career in healthcare'). They could share these Mangomoments with colleagues, inspire them and increase their collaboration and camaraderie. Healthcare professionals and patients report that when Mangomoments are shared within the team, and certainly when you're collaborating to create them, there is an impact on the team atmosphere as it creates or keeps a positive group dynamic.

\section{Loyalty}

In general, Mangomoments increase the loyalty to the organisation. Patients describe that, because of Mangomoments, they tend to come back to receive care in this healthcare organisation and team. Also, healthcare professionals report that they have more feelings of personal accomplishments and joy in work, they will stay more loyal to their organisation. These results suggest that patients and healthcare professionals will become and/or stay ambassadors (see figure 3, red).

\section{DISCUSSION}

In this multicentre multi-method study, 1045 stories were collected of which $94 \%$ were defined as Mangomoments. Mangomoments are the small unexpected acts or gestures, which are of great value in the care experience of patients, residents, families and healthcare professionals. They take place during normal care activities and ask nearly no additional resources, time or energy. Most Mangomoments were included within the dimension 'Respect for values, preferences and needs'. To understand how and when Mangomoments are established and the impact of Mangomoment on patient, family and healthcare professional, 120 semi-structured interviews with patients, families and healthcare providers, 3 focus group meetings and 1 consensus meeting were conducted. From this, the Mangomoment model was developed. Mangomoments have certain preconditions. Catalysts act between these 
preconditions and the Mangomoment itself. When a Mangomoment happens, it can lead to positive experiences for the patient and the family, for the healthcare professional and for the relation between the patient/ family and the professional. The model concludes that the overall outcome of Mangomoments is the loyalty of the patient and family to the healthcare provider and the organisation and of the healthcare professionals to their employer.

Even though Mangomoment was described by a definition and further explained in the Mangomoment model, we noticed that the concept is sometimes confused with patient-centred care. Both concepts clearly differ, but they are interrelated in the sense that Mangomoments are facilitated by a patient-centred culture. Moreover, they give expression to patient-centredness. Not by relying on complex methods, but in a way that can be effortlessly integrated into daily care. Still, there is one clear distinction between both concepts. Patient-centred care is a concept which starts from the perspective of the healthcare professional taking into account the preferences, values and norms of a patient, ${ }^{9}$ whereas Mangomoments can be established in different types of relationships from several perspectives (healthcare professional, patient and management).

Although Mangomoments might not be something spectacular or new, our findings and the energy this study produced in at least 50 healthcare organisations and 30 patient associations in Belgium and The Netherlands, was surprising. Healthcare professionals were enthusiastic for the positive attention to the positive energy for their positive work. They felt that too much attention is given to adverse events and boreout and burnout problems. Therefore this positive project was warmly embraced by clinicians, managers and by (social) media.

The question remains if Mangomoments are the "cherry on the cake' or are one of the fundaments to provide high quality and safe care. In 2011, the Institute for Healthcare Improvement described a model to improve the experiences of patients and their family during hospitalisation. This model contains following five drivers to achieve an exceptional patient and family inpatient hospital experience: (1) Leadership; the culture in the organisation, across the different levels, embraces patient-centred and family-centred care. (2) Heart and minds of the healthcare professional is fully engaged. (3) Respectful partnership, which included that there is a respectful relationship with the patients and they anticipate and respond to patient and family needs. (4) Reliable, quality care is 24/7 available. (5) Evidence-based care is delivered to the patient. ${ }^{10}$ In its work on healthcare improvement across the globe, the Institute for Healthcare Improvement and other global leaders have promoted patient-centredness as a cornerstone of improvement and leadership, one of the six Institute of Medicine domains of healthcare quality, ${ }^{11}$ clearly interconnected with dignity and joy in work. ${ }^{12}$ These are concepts and practices that closely connect with values of love, equity, trust and courage.
Furthermore, these concepts have a mutually beneficial and reinforcing positive effect for patients and healthcare professionals. We have learnt that in leadership it takes courage to let go, to share or cede control to those we serve, and to include patient-centredness and partnership into daily practice in healthcare. And the evidence for leaders is clear: compassion in care is improving patient experiences, decreasing medical errors and is a powerful tool to decreasing costs in healthcare-a sure path to achieving the Triple Aim. ${ }^{13}{ }^{14}$ Mangomoments with their impact on both patients or family and the healthcare professionals can hereby help to enlarge the triple to the quadruple aim, where care for the care provider is the fourth dimension. ${ }^{15}$

In this study an under investigated area in the field of patient safety and quality of care is explored: positive moments in healthcare and its impact on patient, family and healthcare professionals. A limitation is that the researchers did not conduct a member check and that the interviews were not transcribed verbatim, but triangulation methods and peer debriefing with multiple researchers promote validity and credibility of the data. Further research and implementation projects will be launched to further understand the Mangomoment model. The validity of the Mangomoment model will be cross-validated in other cultural contexts. Also, differences between various types of patient groups can help to understand the preconditions and effects. A point of attention is to include neutral and opposite views in follow-up research. Further research should focus on the development of surveys and indicators to follow-up this aspect of person-centred care and benchmark the level of Mangomoment-readiness of teams and organisations. The constructs in the Mangomoment model can be used to develop such surveys. Variation within and between healthcare teams and organisations can help leaders and policymakers to enhance person-centred care initiatives.

Although the six quality dimensions remain a global paradigm, the 'patient' centred dimension was recently redefined into 'person' centred. ${ }^{16}$ Mangomoments are totally in line with this broadening of this concept. If we want to enhance quality and safety, respect toward the patient as a whole person is not the cherry on the cake but the basic fundament that guides every individual act or procedure we perform as a healthcare professional, manager or policymaker. The Mangomoment model explains that optimal collaboration between the triad of patients and family, healthcare professionals and managers and policymakers, is the only way forward. Under these circumstances one can provide an exceptional experience of person-centred care.

\section{CONCLUSION}

Mangomoments are mainly focussed on respecting values, preferences and needs of individual patients. They can have a positive impact but preconditions should be met and there are important influential catalysts on an 
individual and organisational level to have their influence. Mangomoments can have a positive impact on patients and family but also on the joy in work of healthcare professionals. Satisfied patients and happy workers will stay loyal to their healthcare organisation. As Mangomoments have the capability to enhance both aspects, maybe Mangomoments can help us to make healthcare a little sweeter.

\section{Author affiliations}

${ }^{1}$ Leuven Institute for Healthcare Policy - Department of Public Health, KU Leuven University of Leuven, Leuven, Belgium

${ }^{2}$ Department of Quality Management, University Hospitals Leuven, Leuven, Belgium ${ }^{3}$ Department of Critical Care, University Hospitals Leuven, Leuven, Belgium

${ }^{4}$ Medical Department, Jessa Ziekenhuis, Hasselt, Belgium

${ }^{5}$ Department of Urology, University Hospitals Leuven, Leuven, Belgium

${ }^{6}$ Translational Medicine, Amedeo Avogadro University of Eastern Piedmont, Novara, Italy

${ }^{7}$ Institute for Healthcare Improvement, Boston, Massachusetts, USA

${ }^{8}$ Flemish Institute for Quality of Care, Brussels, Belgium

Twitter Kris Vanhaecht @krisvanhaecht, Ellen Coeckelberghs @ellencoeckelberghs, Charlotte Van der Auwera @charlotte Vda, Fien Claessens @Fien Claessens, Luk Bruyneel @lukbruyneel, Dirk De Ridder @dirkjmkdr, Maureen Bisognano @ maureenbis and Pedro L Delgado @okpedrodelgado

Acknowledgements We hereby acknowledge all members of the Stand up for Cancer (Kom Op tegen Kanker) Society for their belief and financial support of this study. We specifically thank Marc Michils, Inge Pelemans, Isabel Weemaes, Lieve Vanderlinden, Lotte Van Looy and Chloë Denayer. We also thank the media team of KU Leuven and all the participating and supporting healthcare organisations and the involved patient associations. We thank Eva Marie Castro for her additional suggestions on patient-centred care and review of the paper.

Contributors Conception and design of the study, KV, MP, DDR, EVB, DS, LB, PLD, MB. Acquisition of the data: EVB, EC, CVdA, FC. Analysis and interpretation of the data: KV, EVB, EC, CVdA, FC, LB, DDR, MP, MB, PLD, DS. All authors critically revised the manuscript for intellectual content and approved the final version.

Funding Kom op tegen Kanker (Stand up to Cancer) funded this project with a grant to the Leuven Institute for Healthcare Policy, KU Leuven. Stand up to Cancer is an NGO that stands up for people with cancer in Flanders.

Patient consent for publication Obtained.

Ethics approval Approval by the University Hospital Leuven ethics committee was obtained (S60022).

Provenance and peer review Not commissioned; externally peer reviewed.

Data availability statement № data are available.

Open access This is an open access article distributed in accordance with the Creative Commons Attribution Non Commercial (CC BY-NC 4.0) license, which permits others to distribute, remix, adapt, build upon this work non-commercially, and license their derivative works on different terms, provided the original work is properly cited, appropriate credit is given, any changes made indicated, and the use is non-commercial. See: http://creativecommons.org/licenses/by-nc/4.0/.

ORCID iDs

Kris Vanhaecht http://orcid.org/0000-0001-5636-4792

Luk Bruyneel http://orcid.org/0000-0003-1209-692X

Deborah Seys http://orcid.org/0000-0003-4966-3879

\section{REFERENCES}

1 Hollnagel E, Wears RL, Braithwaite J. From safety I to safety II: a white paper. The resilient health care net. Australia: The University of Southern Denmark, University of Florida, USA and Marcquarie University, 2015.

2 Fredrickson BL. Love 2.0: finding happiness and health in moments of connection. Hudson Street Press, 2013.

3 Peter P. Patient care: what's love got to do with It? 2016. Available: https://armstronginstituteblogshopkinsmedicineorg/2016/02/12/ patient-care-whats-love-got-to-do-with-it/

4 Vanhaecht K. In search of Mangomoments. Lancet Oncol 2018;19:165.

5 Saunders B, Sim J, Kingstone T, et al. Saturation in qualitative research: exploring its conceptualization and operationalization. Qual Quant 2018;52:1893-907.

6 Gerties M, Edgeman-Levitan S, Delbanco T. Theoretical development of what defines quaity from patient's perspective. San Francisco: Jossey-Bass, 1993.

7 Dierckx De Casterle B, Gastmans C, Bryon E, et al. International Journal of Nursing Studies QUAGOL : A guide for qualitative data analysis. Inter J Nur Stud 2012;49:360-71.

8 Maureen B. Why "what matters to you?" matters around the world, 2017. Available: http://wwwihiorg/communities/blogs/why-whatmatters-to-you-matters-around-the-world

9 Castro EM, Van Regenmortel T, Vanhaecht K, et al. Patient empowerment, patient participation and patient-centeredness in hospital care: a concept analysis based on a literature review. Patient Educ Couns 2016;99:1923-39.

10 Balik B, Conway J, Zipperer L, et al. Achieving an exceptional patient and family experience of inpatient hospital care. IHI innovation series white paper Cambridge. Massachusetts: Institute for healthcare improvement, 2011. www.ihi.org

11 Institute of Medicine. Committee on quality of health care in A. crossing the quality chasm: a new health system for the 21st century. The National Academies Press, 2001.

12 Perlo J, Balik B, Swensen S. Framework for improving joy in work. IHI white paper. Massachusetts: Institute for healthcare improvement, 2017. http://www.ihi.org/

13 Vanhaecht K, Van Bael E, Seys D, et al. Mangomoment: Met Een klein gebaar naar warmere zorg. Acco, 2019.

14 Berwick DM, Nolan TW, Whittington J. The triple aim: care, health, and cost. Health Aff 2008;27:759-69.

15 Bodenheimer T, Sinsky C. From triple to quadruple aim: care of the patient requires care of the provider. Ann Fam Med 2014;12:573-6.

16 National Academies of Sciences E, and Medicine. Crossing the global quality chasm: improving health care worldwide. Washington DC: The National Academies Press, 2018. 